\title{
Alternatives to direct emergency department conveyance of ambulance patients: a scoping review of the evidence
}

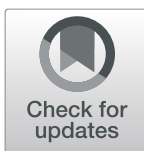

\author{
Joanna M. Blodgett ${ }^{1,2,3^{*}}$ D, Duncan J. Robertson ${ }^{2,4}$, Elspeth Pennington ${ }^{2}$, David Ratcliffe ${ }^{2,5}$ and Kenneth Rockwood ${ }^{3}$
}

\begin{abstract}
Background: The role of ambulance services is shifting, due in part to more intermediate, non-urgent patients who do not require direct emergency department conveyance, yet who cannot be safely left at home alone. Evidence surrounding the safety, effectiveness and efficiency of alternate care routes is not well known.

Methods: This scoping review sought to identify all studies that examined alternate routes of care for the nonurgent "intermediate" patient, as triaged on scene. Search terms for the sample (ambulances, paramedics, etc.) and intervention (e.g. referrals, alternate care route, non-conveyance) were combined. Articles were systematically searched using four databases and grey literature sources (February 2020). Independent researchers screened titleabstract and full text stages.

Results: Of 16,037 records, 41 examined alternate routes of care after triage by the on-scene paramedic. Eighteen articles considered quantitative patient data, 12 studies provided qualitative perspectives while 11 were consensus or opinion-based articles. The benefits of alternative schemes are well-recognised by patients, paramedics and stakeholders and there is supporting evidence for a positive impact on patient-centered care and operational efficiency. Challenges to successful use of schemes included: patient safety resulting from incorrect triage decisions, inadequate training, lack of formal partnerships between ambulance and supporting services, and insufficient evidence to support safe implementation or continued use. Studies often inaccurately defined success using proxies for patient safety (e.g. decision comparisons, rates of secondary contact). Finally, patients expressed willingness for such schemes but their preference must be better understood.

Conclusions: This broad summary offers initial support for alternate routes of care for intermediate, non-urgent patients. Even so, most studies lacked methodologically rigorous evidence and failed to evaluate safe patient outcomes. Some remedies appear to be available such as formal triage pathways, targeted training and organisational support, however there is an urgent need for more research and dissemination in this area.
\end{abstract}

Keywords: Ambulance, Alternative care routes, Non-emergency medical care, Pre-hospital emergency care, Scoping review, Referrals

\footnotetext{
* Correspondence: Joanna.blodgett.16@ucl.ac.uk

'MRC Unit for Lifelong Health and Ageing, UCL, 1-19 Torrington Place,

London WC1E 7HB, UK

${ }^{2}$ North West Ambulance Service, NHS Trust, Bolton, UK

Full list of author information is available at the end of the article
}

C C The Author(s). 2021 Open Access This article is licensed under a Creative Commons Attribution 4.0 International License, which permits use, sharing, adaptation, distribution and reproduction in any medium or format, as long as you give appropriate credit to the original author(s) and the source, provide a link to the Creative Commons licence, and indicate if changes were made. The images or other third party material in this article are included in the article's Creative Commons licence, unless indicated otherwise in a credit line to the material. If material is not included in the article's Creative Commons licence and your intended use is not permitted by statutory regulation or exceeds the permitted use, you will need to obtain permission directly from the copyright holder. To view a copy of this licence, visit http://creativecommons.org/licenses/by/4.0/ The Creative Commons Public Domain Dedication waiver (http://creativecommons.org/publicdomain/zero/1.0/) applies to the data made available in this article, unless otherwise stated in a credit line to the data. 


\section{Introduction}

Ambulance trusts continue to experience annual increases in the number of emergency calls. In 2000, the UK Ambulance services responded to 4.41 million calls per year [1]. By 2018-2019, call frequency had more than tripled, reaching a record high of 13.8 million calls [2-5]. However, a large proportion of these emergency calls do not require emergency department (ED) attendance $[6,7]$. While some patients can be safely left at home, many others are conveyed to hospital for nonurgent care and contribute to ED overcrowding [8]. Limitations in ED capacity can lead to long delays in corridor wait time, reduced availability of ambulances for subsequent emergencies and rising health care costs [911].

While 999 dispatch processes aim to triage patients at the point of call, it can be difficult to ascertain what a patient needs until there is a healthcare practitioner on scene. When on scene, paramedics often triage the patient to determine necessity of direct ED conveyance or if it is safe for the patient to be left at home [12]. Even so, this leaves an important group of "intermediate" patients, who now are the driving force behind increased ED attendance. There is no clear definition of such patients, although the UK Paramedic Pathfinder triage system defines these patients as amber, while the Swedish Rapid Emergency Triage and Treatment System (RETT S) triages them as yellow or green. Based on these objective definitions, we define "intermediate" patients as those with non-urgent medical illnesses or injuries who may not require conveyance to the $\mathrm{ED}$, yet cannot be safely left at home without medical support [13]. Due to a lack of access to alternative routes of care, these intermediate patients are often unnecessarily conveyed to ED [14]. Non-urgent patients account for up to $50 \%$ of all ED attendances $[15,16]$, suggesting that there is a need for ambulance services to target alternative routes of care for such patients.

At both the national and international level, there is no consensus on alternative routes of care to direct ED conveyance. With a distinct lack of empirical evidence, a collaborative effort across ambulance trusts is required to identify potential benefits or consequences for the individual and the healthcare system as a whole [17]. Older systematic reviews document few alternative care pathways and insufficient evidence to deduce whether they are safe [18]. Jensen et al. [19] recently catalogued outcomes of alternative emergency medical services (EMS) dispatch and transportation programs but, to our knowledge, there is no consensus understanding of the protocols of such schemes nor of the supporting evidence. Interventions that allow paramedics to appropriately direct individuals to alternative care pathways can ensure patient safety, improve ambulance and ED efficiency, whilst also providing substantial savings to the healthcare system [20] .

As different ambulance trusts in varying countries begin to explore alternative routes of care for these intermediate patients, synthesis of current programs, services and protocols is crucial. To avoid ambulance services working in isolation, it is important to recognize what different services have implemented, and whether or not these services are safe and beneficial to the patient and the health care system. Given the lack of evidence in this area, this scoping review had the following objectives:

1) to identify all studies that examined alternatives to direct ED conveyance for patients triaged by the on-scene emergency medical clinician;

2) to describe all alternative schemes and study outcomes in the identified studies and;

3) to assess the quality of the evidence provided.

Due to our prior research in this field [16], we hypothesised that there would be strong heterogeneity of alternative non-ED schemes across various emergency medical services, a low quality of evidence and limited evidence that adequately assessed patient outcomes.

\section{Methods}

Scoping reviews are used to map the key concepts that constitute the groundwork of a specific area as well as the main source and type of evidence available [21]. Here, we aim to attain a representative and nearcomprehensive sample of the evidence in a pre-defined topic area and to describe the quality of the evidence base available in terms of study design and questions addressed by the identified studies [22]. The methodology of this review follows the Preferred Reporting Items for Systematic Reviews and Meta-Analyses extension for Scoping Reviews (PRISMA-ScR) statement [23].

\section{Search strategy}

We used initial pilot searching in two databases to aid in defining effective search terms (JB, DR, additional librarian support). This iterative process provided confidence that the search strategy was adequately capturing all relevant studies. The resulting search strategy remained purposely broad and thus the numbers of studies captured by the search was expected to be large. The initial search strategy was conducted in April 2016 but was updated in April 2017 and February 2020. We searched PubMed, CINAHL, Web of Science and ProQuest Health \& Medicine databases. Two separate arms were combined using the Boolean operator "AND" to search for the "who" (paramedic or ambulance) and the "what" (referral or non-conveyance). The full search strategy 
can be found in Appendix; it was adapted as necessary for each additional database. To ensure all relevant studies were adequately captured, no exclusion filters were applied for language, year (e.g. inception to February 2020) or article type. We also searched grey literature on the following sites: NHS Evidence, CORE, BL.UK, OpenGrey, and HMIC. Additional articles were found by a snowball search of the reference lists of relevant systematic reviews and of articles that met the inclusion criteria.

\section{Study selection}

In the first stage of screening, two researchers independently screened the title and abstracts of each article for relevancy (JB, DR, EP). The second stage of screening involved retrieval and evaluation of the full text to identify if it met the criteria. Again, two of these three researchers independently screened the full text of all potential studies. Reviewers discussed any discrepancies and where necessary, a third reviewer made the final decision. Reason for exclusion in the full text stage was noted.

Inclusion criteria purposely remained broad to capture relevant studies. Articles were included if they considered emergency service callers (e.g. 999, 911, etc.) who were triaged by the on-scene clinician as non-urgent "intermediate" patients and if there was an indication of an alternate route of care to conveyance to ED. "Intermediate" patients were those with non-urgent medical illnesses or injuries who did not require conveyance to the ED, yet could not be safely left at home without medical support [13]. Emergency department is defined as a medical facility responsible for treatment of patients who arrive at the hospital and require immediate medical care. Undifferentiated "intermediate" patients who did not fit within an existing evidence-based pathway were included, while samples of specific clinical patients with pre-existing evidence-based pathways were excluded (e.g. falls, resolved hypoglycaemia, psychiatric and resolved epilepsy pathways). Commentaries, protocols and policy statements were eligible for inclusion, while literature reviews, conference abstract, nonEnglish and non-peer reviewed articles were excluded from final article selection. Due to the expected heterogeneity of studies, there were no limitations on study outcomes. The following software were used in study selection and screening: Mendeley, Microsoft Excel and Rayyan.

\section{Data extraction}

JB independently extracted data from all studies using a standardised form that was agreed upon and piloted by the research team (JB, DR, EP). This included data on country, study type, sample size, description of alternate route of care, triage protocol, study outcomes and study findings. A meta-analysis was not possible due to expected heterogeneity of included studies, and a narrative synthesis was conducted instead. The heterogeneity in design, methods and outcomes of studies also facilitated three groups for synthesis: 1) quantitative patientfocused, evidence-based studies; 2) qualitative, evidencebased studies; and 3) consensus-based articles.

In line with the second objective, two authors (JB, EP) independently appraised the quality of evidence following a modified 7-level rating system for the hierarchy of evidence [24]. This tool provides a hierarchy of the likely best evidence (e.g. Levels 1 to 7) and is specifically designed to aid clinicians (and patients) with a rapid appraisal to avoid the need to resort to original sources. Differences in levels of evidence between authors were discussed and agreed upon in a consultative process.

\section{Results}

The search revealed 15,968 single records from databases and 69 from grey literature and reference list screening. After title and abstract screening, 383 records remained for the full text screening stage. Most articles were excluded as they did not mention an alternate route of care $(n=105)$, focused on ED related issues $(n=64)$ or other public health initiatives $(n=65)$ including pre-ambulance care, walk-in primary care, community paramedicine, air ambulance or other emergency professions (firefighting, police). Other exclusions were for specific clinical problems $(n=36)$, diversion to other emergency hospital facilities $(n=18)$, triage related $(n=$ $24)$ or wrong article type $(n=30)$. Of the 41 included studies, there were 18 quantitative evidence-based studies, 12 qualitative evidence-based studies and 11 consensus-based articles including commentaries, protocols and policies. A PRISMA flow-diagram outlining study screening and selection is shown in Fig. 1.

Levels of evidence of all 41 studies were graded from 1 to 7 , as described above [24]. Of the 18 quantitative studies, two were graded as a Level 2 due to their random allocation of intervention and control groups, 11 studies were graded as Level 4, four studies as Level 3 and a single descriptive study was graded as a Level 6 . All 12 qualitative studies were graded as a 6; while they demonstrated perceived support for such schemes from individuals involved, they did not contribute evidence to understanding if the scheme is safe and effective. Finally, all consensus-based papers were graded as a 7 (i.e. expert opinion), given that they did not contribute any evidence to the field.

\section{Quantitative, evidence based studies}

Eighteen studies with patient-focused, quantitative evidence are outlined in Table 1 . Seven studies were based 


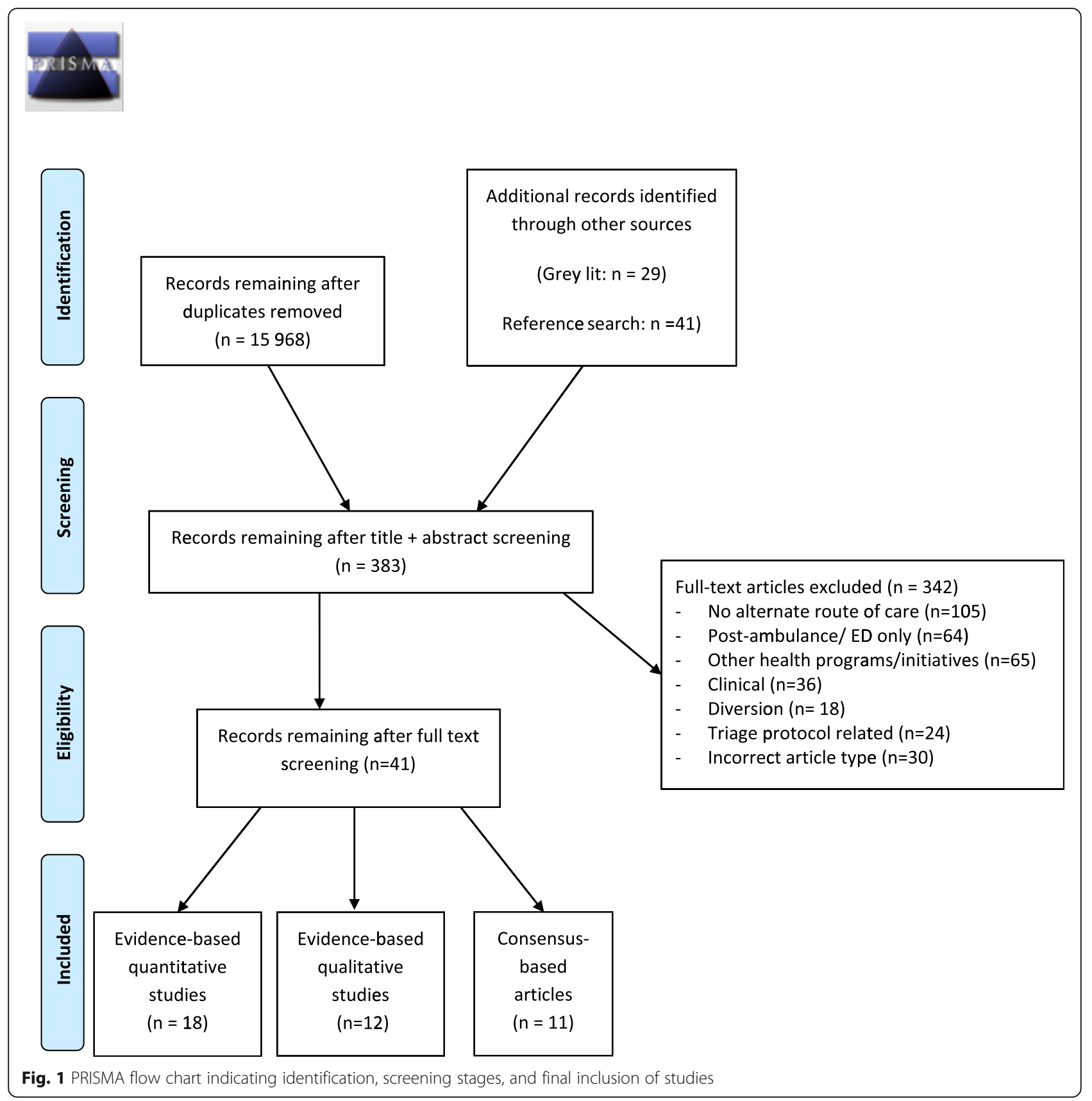

in the UK, four in both the USA and Sweden and one in each of Canada, Australia and the Netherlands. Only five studies compared an intervention group (e.g. option to refer to alternate care route) and a control group (e.g. normal practice), with two of these studies randomising into either arm [37, 41]. Retrospective cohorts, including data audits, were the most common study design $(n=8)$, followed by prospective cohorts $(n=7)$ and randomised control trials (RCTs) $(n=2)$. One study used a comprehensive mixed-methods approach of interviews, qualitative telephone data and linked retrospective data [32].
Most studies $(n=14)$ outlined a triage protocol to guide the ambulance clinician's decision making; this included six studies with triage tools that led directly to alternate care route outcomes, five studies with a series of protocols for specific incidents and three studies that allowed subjective referral of patients triaged as low acuity using traditional triage tools. Studies commonly detailed multiple alternative care routes that could be accessed through either ambulance transport or referral. Referrals to primary care, including general practitioners (GPs) or nurses, were most frequent $(n=16)$, although options 


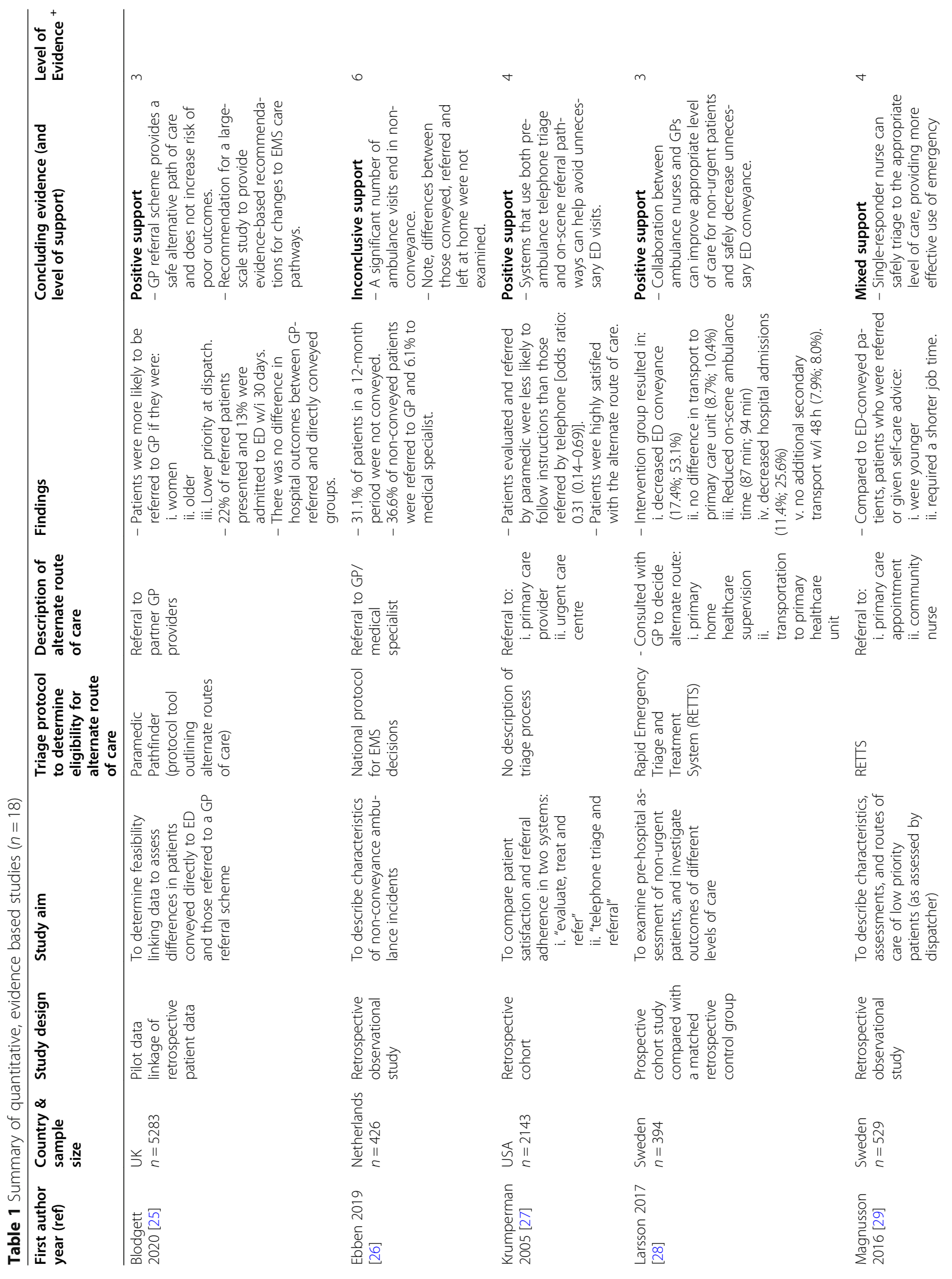




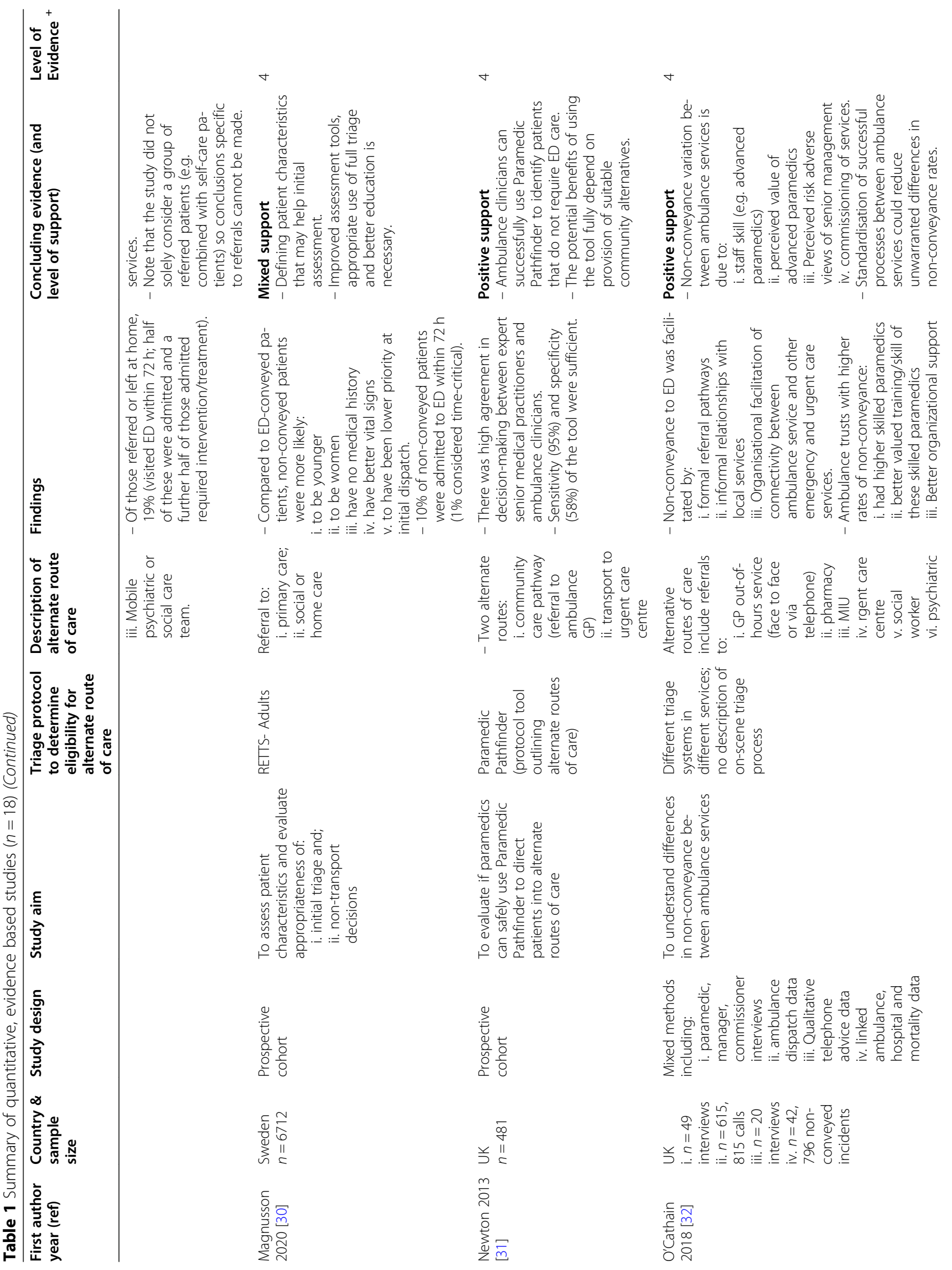




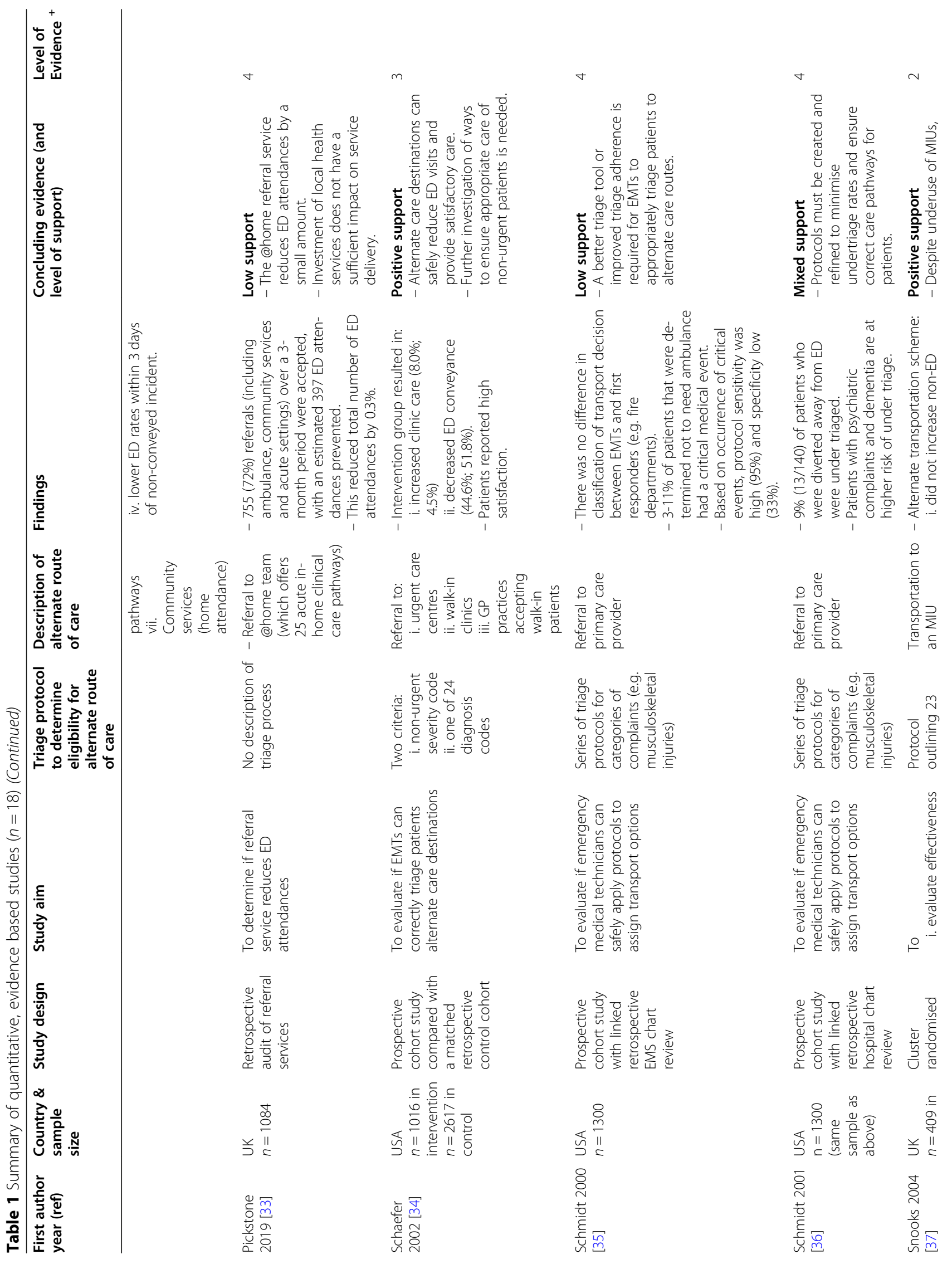




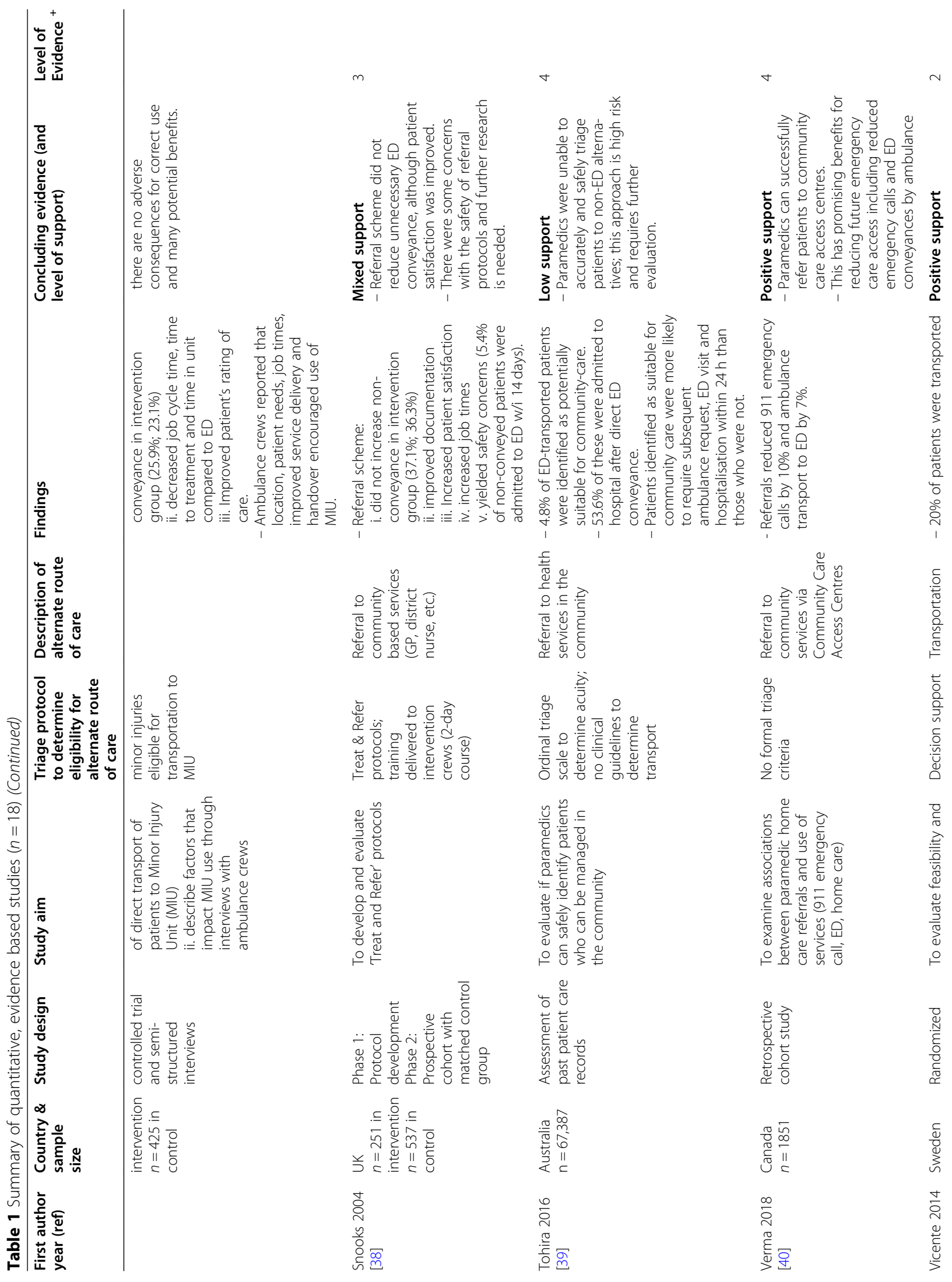




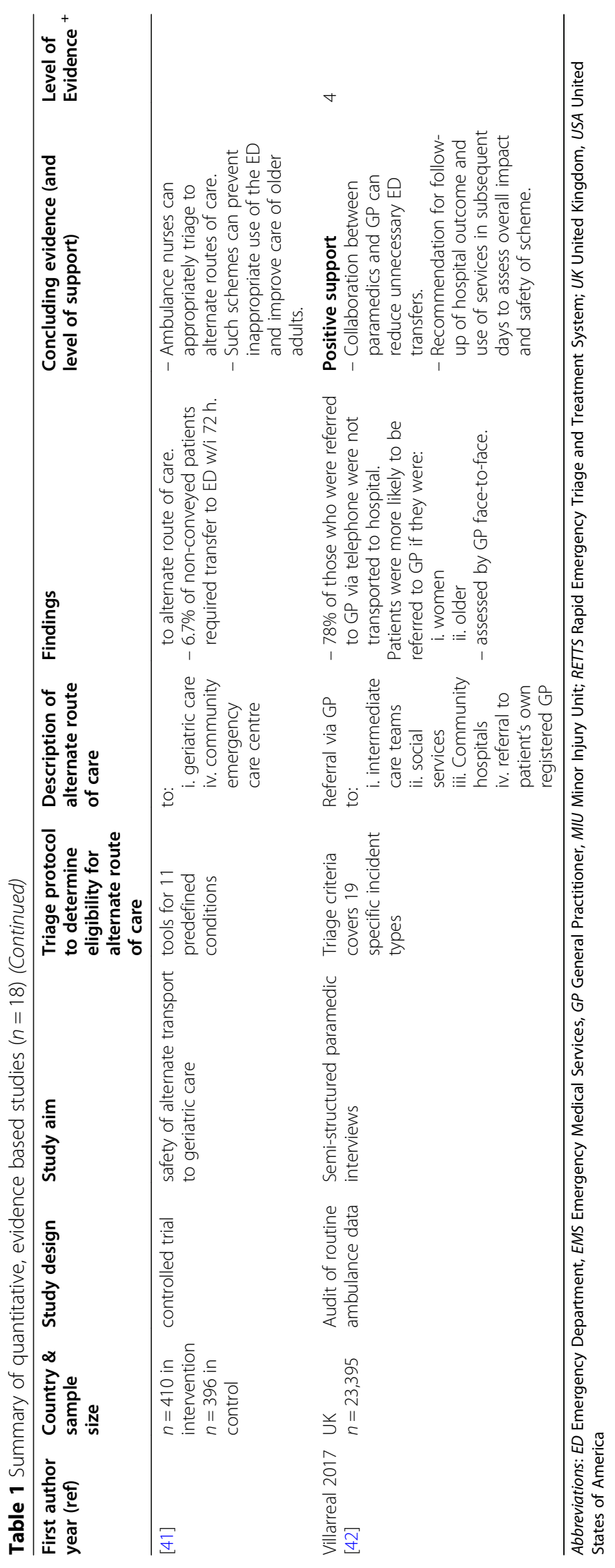


such as urgent care centres $(n=6)$, psychiatric or social teams $(n=4)$ and minor injury units $(n=2)$ were also common.

We identified two main themes: patient safety and impact on operational efficiency. Most studies suggested that paramedics were able to accurately triage patients to the correct pathway of care [31, 32, 35-37, 40, 41]. For example, several studies compared decisions between paramedics, first responders (e.g. fire departments) and senior medical experts reporting high sensitivity $(>94 \%)$ and lower specificity $(<58 \%)[31,35$, $36]$. Rates of secondary contact with emergency medical services (e.g. calls, transport, ED presentation) were reported in six studies (Fig. 2). Secondary presentation at ED occurred in 5.4-22.4\% of all patients who were referred to an alternate care route (range: $48 \mathrm{~h}$ to 30 days) $[25,28-30,38,41]$; approximately half were subsequently admitted $[25,29,30]$. Only two studies compared rates in intervention and control group, reporting no difference in secondary ED presentation $(7.9 \%$ vs $8.0 \%$ ) [28] or admission rates (5.4\% vs $6.2 \%$ ) [38]. Not all studies reported that paramedics could correctly triage patients. For example, one study reported that $53.6 \%$ of patients identified by paramedics as eligible for alternative routes of care were subsequently admitted to hospital and were also more likely to experience adverse events than those who conveyed directly to ED [39]. Other studies highlighted concerns of under triage as shown by high secondary recontact rates, overturned decisions by medical experts $(19.5-22 \%)$ [31, 42] and occurrence of critical events in those incorrectly triaged away from ED (3-11\%) [35, 36]. Psychiatric presentations and patients living with dementia were considered at particular risk of under triage [36].

Studies reported that alternative care routes improved operational efficiency by decreasing ambulance job cycle times $[28,29,37]$, decreasing ED conveyance rates [26, $28,32,34,40,41]$, improving patient documentation [38], increasing clinic care destinations [34], decreasing hospital admissions $[25,28,33]$ and improving or maintaining patient satisfaction [31, 34, 37, 38]. Nevertheless, there were conflicting findings that reported longer job cycle times [38] or showed that alternative care schemes had no impact on decreasing ED conveyance rates [37, 38].

\section{Qualitative, evidence based studies}

Characteristics of the 12 qualitative studies are provided in Table 2. Study characteristics of the qualitative studies were similar to above, with a third of studies $(n=4)$ in each of the UK and Sweden, two studies in Ireland and one in each of Canada and the USA. As in the quantitative studies, the main alternate care routes comprised of primary care referrals, referral or transport to urgent or community care centres and transport to minor injury units. Data was primarily collected from interviews with

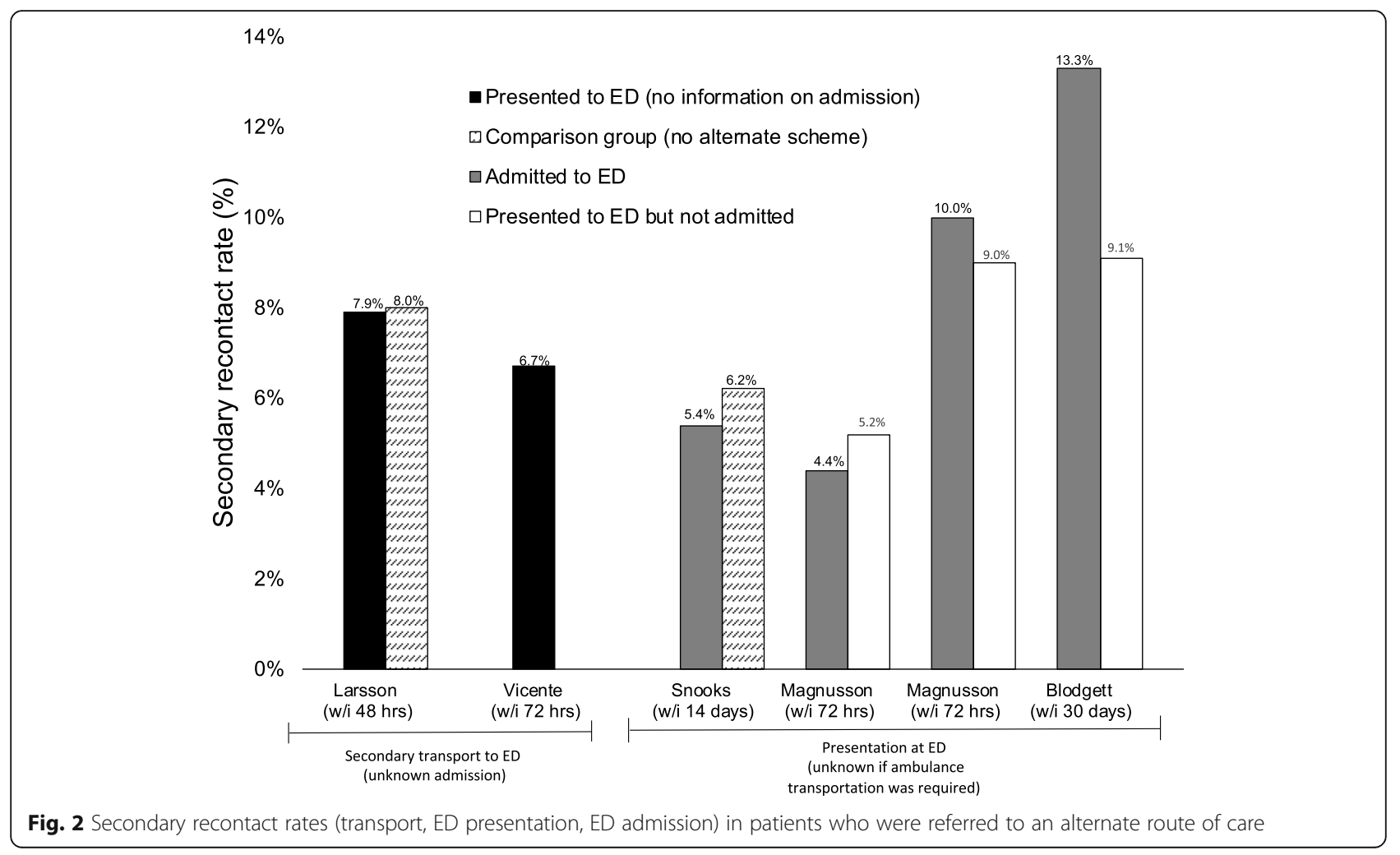




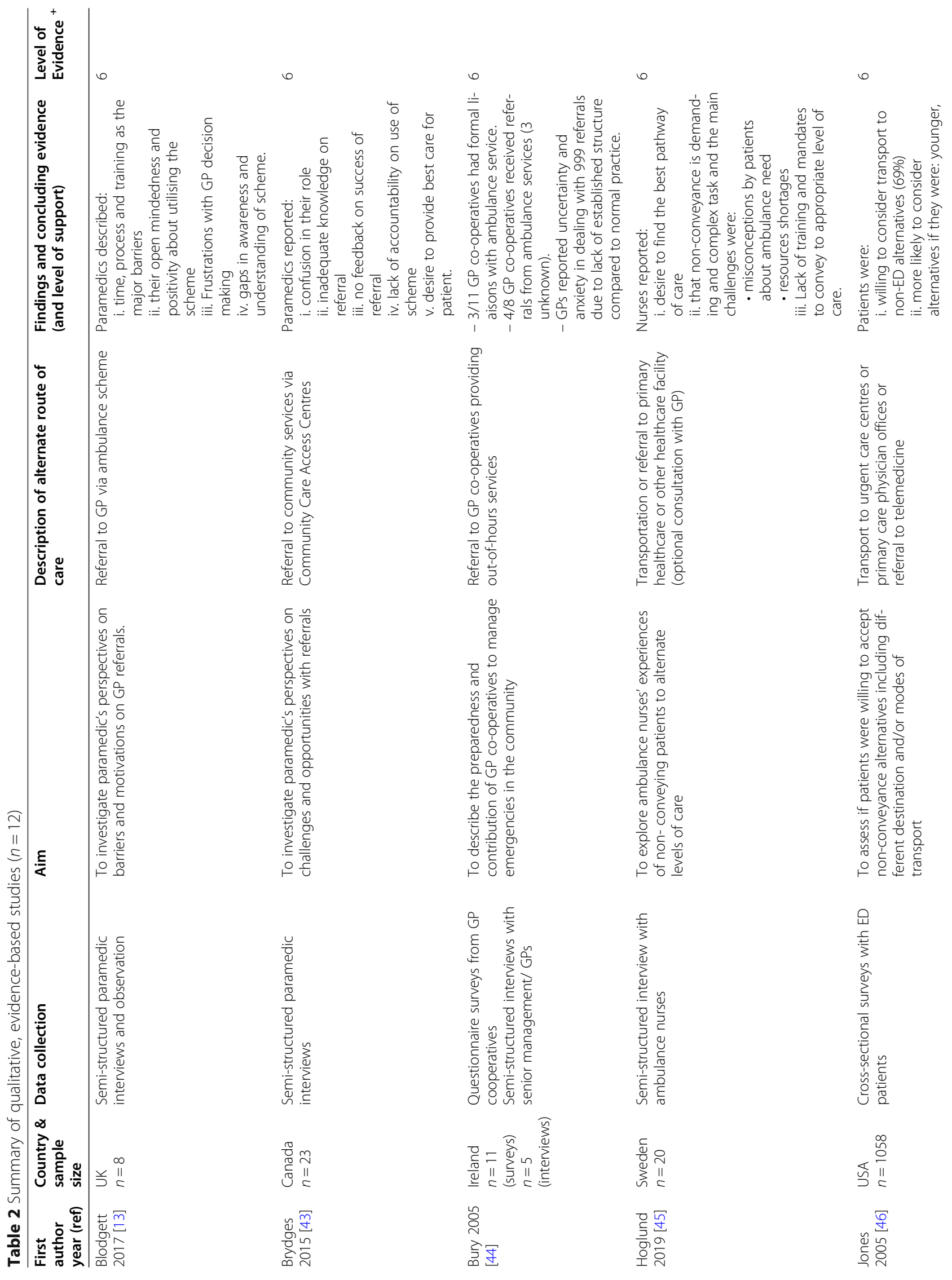




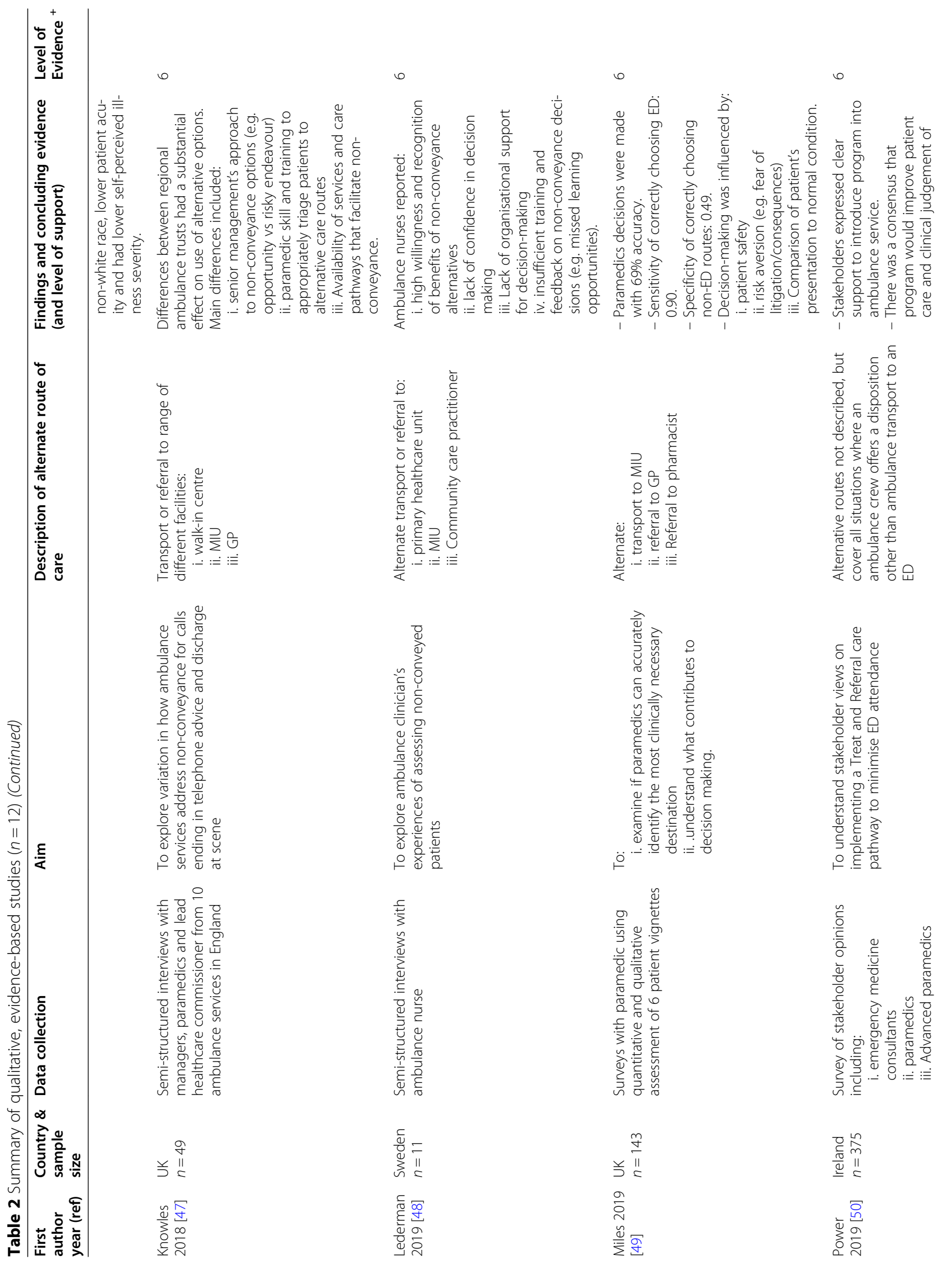




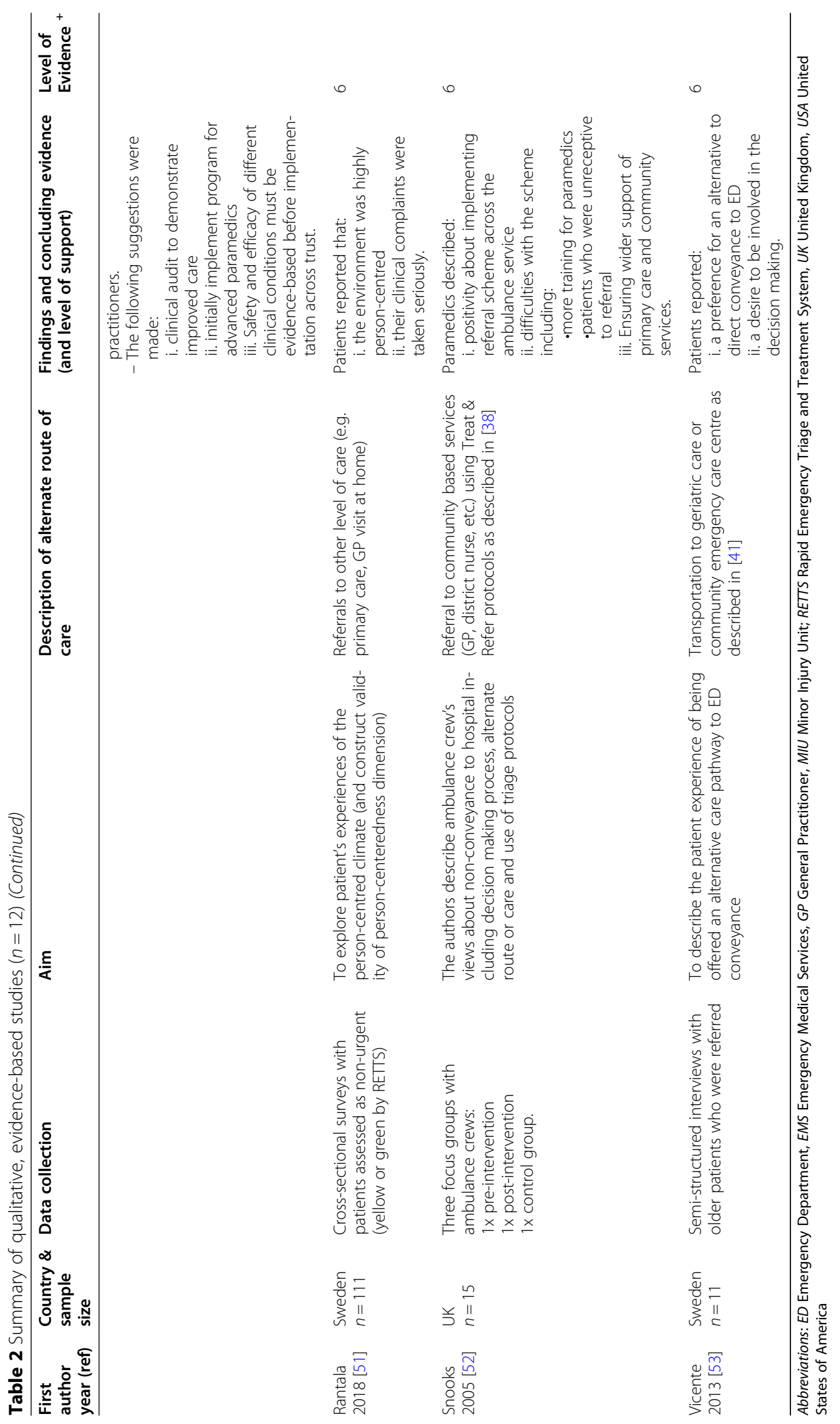


paramedics $(n=6)$ or ambulance nurses $(n=2)$, patients $(n=3)$, emergency medical physicians $(n=2)$ or senior managers/commissioners of ambulance trusts $(n=2)$. There was unanimous support from all studies that schemes providing alternate routes of care than direct ED-conveyance can deliver vast benefits, although several barriers were recurrently identified.

The main barriers identified by paramedics to successful implementation were training, organisational support and process. First, the absence of training and knowledge on referral triage and processes $[13,43,45,47,48$, 52] often manifested as a lack of confidence in both themselves and the system [13, 48], with some paramedics expressing concerns about being held accountable or facing legal action for under triaging patients $[47,49]$. Next, there were concerns that the absence of a mandate from their organisation led to a lack of responsibility to refer and, thus, underuse of the scheme [43, 45]. Organisational support from ambulance services and partnerships with primary or community care pathways were identified as crucial components of an effective scheme [45, 47, 48, 52]. Paramedics did not receive feedback on patient outcomes from their own organisation or the referral destination; they reflected that this was demotivating and a lost learning opportunity [43, 48]. Finally, although uncommon, paramedics expressed minor frustrations about the referral process itself [13], perceived time spent to refer [13, 43] and misconceptions from patients who wanted direct conveyance to ED $[45,52]$.

Consistent with views from paramedics, interviews with stakeholders identified the importance of established structures between organisations [44, 47] and sufficient training and knowledge [50] of paramedics. Power et al. [50] was the sole paper to contrast viewpoints between emergency medical consultants and paramedic towards "treat and referral" schemes. Emergency medical consultants more commonly reported that referral/transport to ED alternatives should be limited to advanced paramedics $(57.2 \%$ vs $22.6 \% ; p<0.001)$, that paramedics should inform the patient's GP after each referral/alternate transport $(88.9 \%$ vs $47.5 \%)$ and were less optimistic that the scheme would improve ambulance availability ( $55.6 \%$ vs $83.9 \%$ ) [50]. Finally, patients expressed both a willingness [46] and a preference [53] to be directed to an alternate scheme rather than conveyed directly to ED. They emphasised their desire to contribute to the decision-making process [53], and felt that their views were taken seriously $[51,53]$.

\section{Consensus- based studies}

Six of the 10 consensus-based articles were based on the American EMS systems, three concerning UK systems and one on Australian systems. These articles were primarily opinion-based ( $n=2$ editorials, $\mathrm{n}=2$ viewpoints, $n=3$ commentaries), but also included two policy statements, a scheme overview and one promising RCT study protocol. Details of all studies are provided in Table 3. Note that results from the study protocol are not publishable due to "unresolvable inconsistencies in data" [57]. The opinion-based pieces and the policy statements both highlighted the promising potential for such schemes, with particular emphasis on projected cost savings [54, 58, 62, 63]. A policy statement, written in 2001 by the Emergency Medical Services Committee and reaffirmed in 2008 by the American College of Emergency Physicians, highlighted seven key elements that should be considered when implementing alternative routes of care $[59,60]$ (see Table 3). Consistent with above, patient safety and accuracy of paramedic triage decisions were raised as main areas of concern [16, 58, 63, 64]. Sawyer et al. [64] was the sole article to recommend against implementation of such schemes citing concerns of insufficient supporting evidence, under triage having an adverse effect on patient safety and vulnerable patients being disproportionally affected.

\section{Discussion}

This broad scoping review provided an overview of 41 articles examining alternate routes of care to direct ED conveyance. Despite heterogeneous study characteristics and diverse alternative care pathways, there was strong consensus from patients, paramedics and other healthcare practitioners of the benefits of alternative care schemes. Even so, several key barriers were emphasised. Positive support broadly covered three topics. First, nonED alternatives were reported to improve operational efficiency by decreasing ED conveyance, reducing incident time and providing projected savings across the emergency health care sector. Next, there was clear recognition by paramedics, stakeholders and patients that alternate care schemes can provide optimal patient care pathways. Finally, despite unanimous recognition of the importance of patient safety when using or implementing these schemes, there was mixed evidence as to whether paramedics could accurately and safely triage patients to the appropriate level of care. Other barriers included insufficient evidence of patient safety, unsatisfactory training, and a lack of formal partnerships between ambulance services and supporting services.

Recommendations for a successful alternate route of care Analysis of patient data and interviews with those involved in the care pathway (e.g. patient, ambulance clinician, stakeholder) suggested that successful schemes share four key features. First, clear triage tools are crucial in guiding accurate decision making of ambulance 


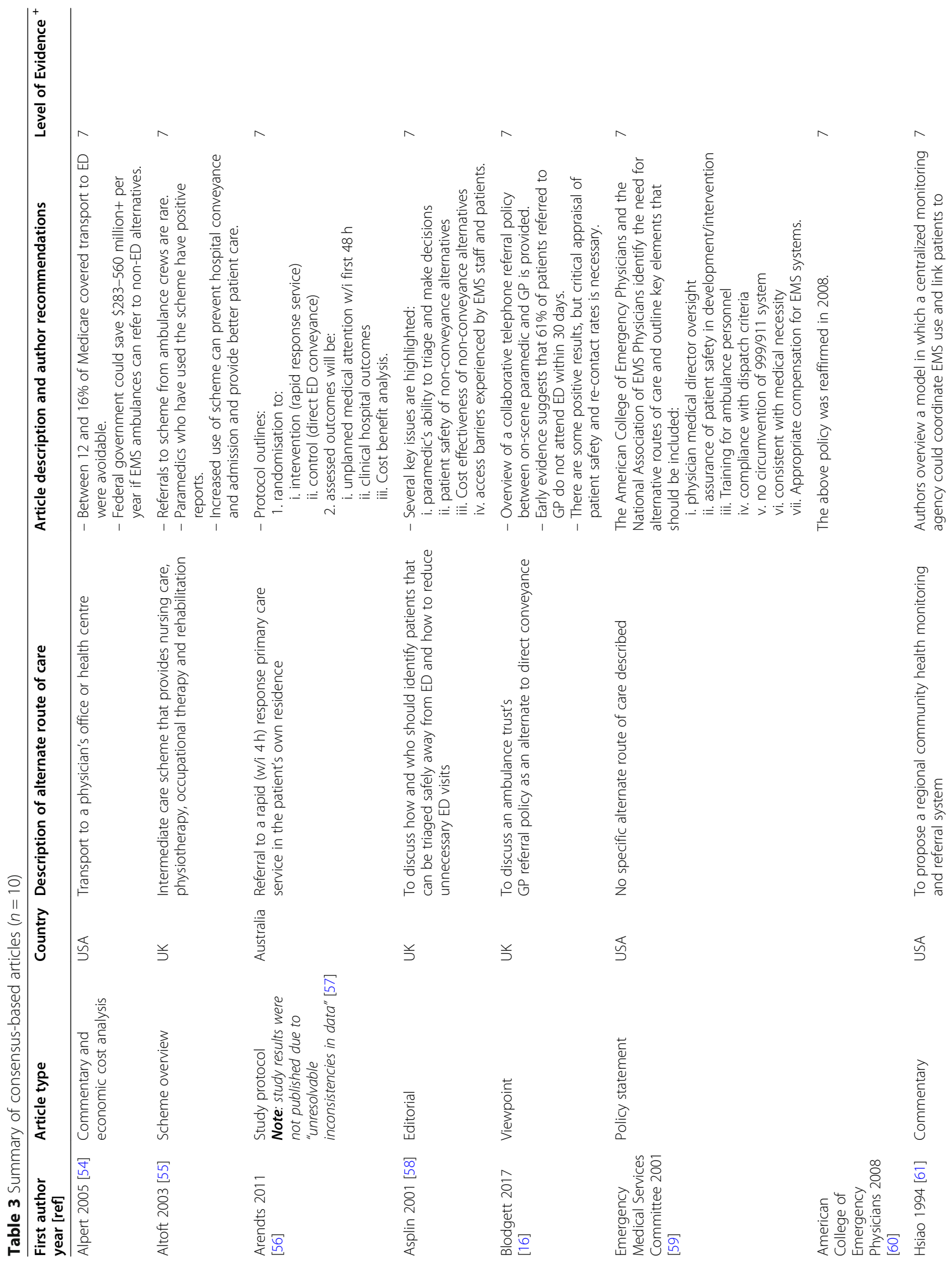


Blodgett et al. Scandinavian Journal of Trauma, Resuscitation and Emergency Medicine

(2021) $29: 4$

Page 16 of 21

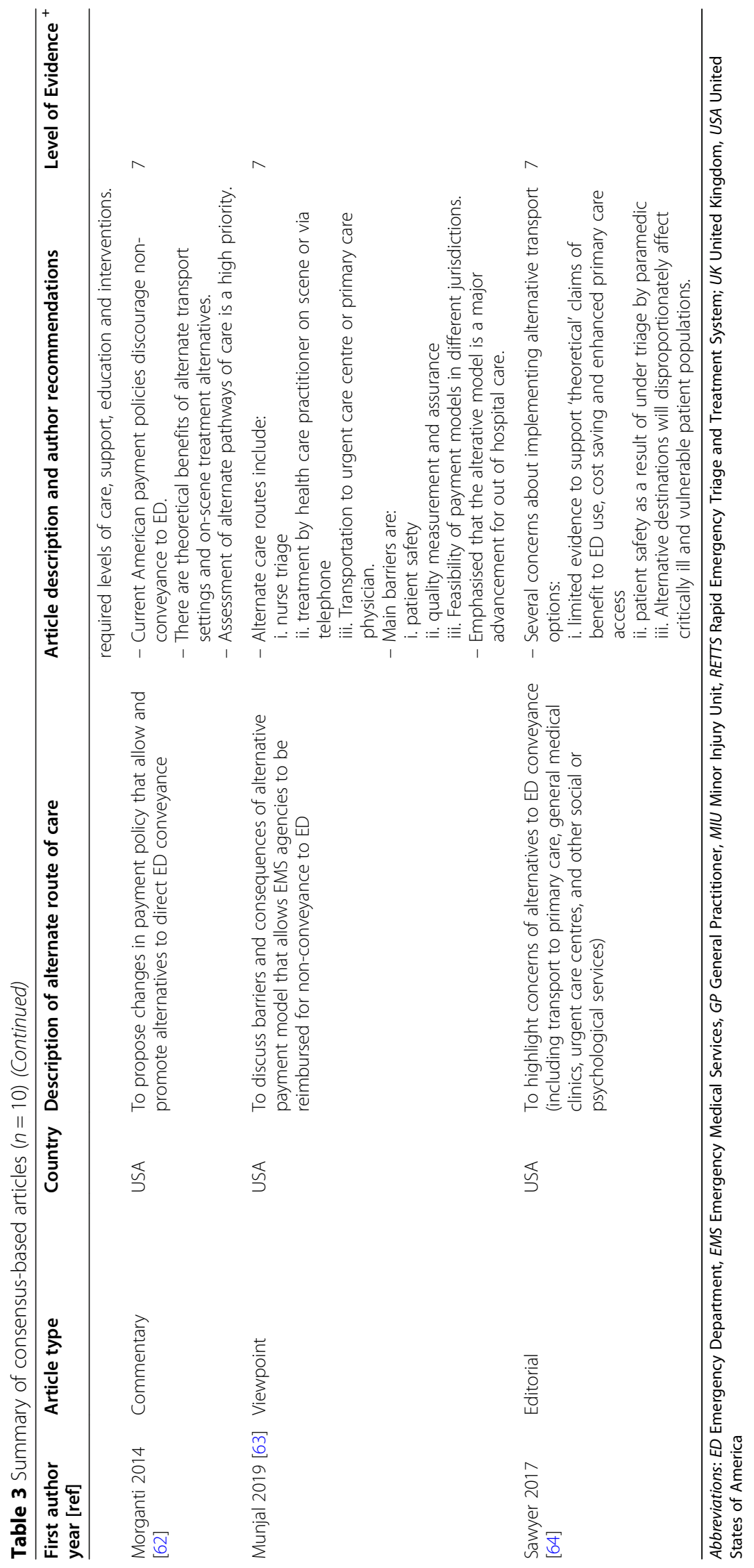


clinicians. Formal triage pathways can ensure that patients are referred or conveyed to the appropriate destination. For example, tools such as the UK-based Paramedic Pathfinder [31] or the Swedish-based Rapid Emergency Triage and Treatment System (RETTS) [2830] allow triage outcomes that direct patients to a specific alternative care route (e.g. GP referral, community care centre). Without a guiding framework, non-specific triage tools can lead to incorrect triage decisions [34, 39]. There is no universally accepted triage tool that could be applied everywhere as the availability of schemes differs by health care system, both within and across country. Additionally, geographical considerations play an important role as densely populated urban areas may be able to facilitate non-conveyance of patients better than rural areas with fewer resources. As such, formal triage tools must be specific to individual healthcare systems and provide clear support to guide the decision making of ambulance clinicians.

Second, additional training on correct use of alternative care schemes including triage tools, overview of processes and learned examples is necessary. Although higher skilled ambulance clinicians (e.g. advanced paramedics, ambulance nurses) may have improved decisionmaking processes, there was a persistent belief that paramedics of all skill levels should still be trained to appropriately use such schemes. Inadequate training likely explains why paramedics have perceived low confidence and hesitation to use these schemes [43, 48, 52].

Third, formal liaisons and partnerships between ambulance services, primary care, urgent care centres, minor injury units or psychiatric and social teams are crucial in facilitating referral or alternate transportation of patients. Without well-established pathways of care, ambulance clinicians are forced to rely on ad hoc decisions and, as a result, are often unsuccessful in finding an appropriate alternative source of care [32]. This must be a consideration when considering implementation of schemes in different countries or regionalised ambulance services, which may limit availability of alternate facilities. Finally, the most important and persistent recommendation in quantitative, qualitative and consensusbased studies was the need for adequate evidence demonstrating patient safety. It was commonly suggested that the current evidence was not sufficient to justify implementation of such schemes; this is described further below.

\section{Ongoing concerns and challenges}

Research in this area is increasing, contributing to and reflecting positive developments in the paramedicine profession [65, 66]. Half of the studies were published within the last 5 years, with only one study coming before the turn of the century [61]. Expert opinions and viewpoints identify the importance of providing alternatives to direct ED conveyance, while qualitative studies are fundamental in describing the views of ambulance clinicians to understand how human factors can ensure optimal use of these schemes [67]. However, the quality of existing evidence is poor, particularly as it pertains to the most important outcome of patient safety.

The overall level of evidence is low, with only two studies using an RCT design (Level 2) [37, 41]. RCTs are essential to assess the impact of an intervention on patient outcomes [68]. Here, they enable alternative care schemes to be compared to ambulance services without alternative options and may provide a holistic overview of patient outcome differences. Conversely, nearly all relevant studies failed to formally assess whether the schemes are safe and instead, considered accuracy of conveyance decisions (e.g. between ambulance clinicians and expert medical consultants) [31, 35, 36, 49] or rates of secondary contact $[25,28-30,38,39,41]$ as proxies for patient safety. While accuracy of decision making may inform future outcomes, it is important to consider both short and long-term patient safety. Secondary contact rates may not provide a reliable indication of individual outcomes. For example, patients may contact emergency services for a reason unrelated to the original incident or recontact rates may be inaccurate if data from all possible health services (e.g. GP, ambulance, $E D$, etc.) are not obtained. Additional secondary contact with emergency services is common regardless of patient destination; only one study considered how recontact rates in a clear intervention group (e.g. with non-ED conveyance alternatives) compared to a control group, reporting there was no difference in secondary transport within $48 \mathrm{~h}$ ( $7.9 \%$ vs $8.0 \%)$ [28]. Failure of studies to appropriately consider the impact on the whole system has previously been highlighted [49].

It can be challenging to generalise findings across countries or between services in the same country, as the structure of the EMS system poses unique challenges. For example, there may be higher potential for alternative schemes in the publicly funded UK system which may allow better linkage within healthcare facilities, while privately funded and delivered health care systems, such as the USA, may encounter different challenges $[19,69,70]$. Similarly, Swedish ambulances are staffed with qualified nurses, which may provide them with a larger scope of practice in their decision making than emergency medical technicians in other countries $[71,72]$. Most articles proposing cost efficiency of these schemes are US-based and consider specific regional EMS organisations and privatized primary care; this makes it difficult to draw conclusions for a federally funded healthcare system. 
Finally, it is important to identify an acceptable under triage rate in non-ED conveyance situations [10]. High sensitivity of tools suggest that ambulance clinicians are able to accurately assess who needs to go to ED, while the lower specificity indicates that they are less able to identify who may benefit from an alternative care route $[31,35,49]$. Over conveyance to ED is preferable to a model that regularly under triages individuals to lower levels of care than is required, and as such, triage tools for non-ED alternatives may necessitate a highsensitivity, low specificity approach [10]. Further evaluation and assessment of the safest level of under triage requires further investigation.

\section{Limitations}

Due to the heterogeneity of the literature, the identification of relevant articles was challenging; despite a wide and inclusive search strategy, it is possible that relevant articles were not identified. Furthermore, due to ongoing innovation of the paramedicine field, we believe that there is a significant amount of discourse on alternate routes of care that has not been formally researched or published. It is important to disseminate results that have undergone formal peer-review to assure the highest quality of evidence and to help establish the evidence base. Many ambulance services work in isolation as they navigate and identify these alternative care pathways; communication between services both within and between countries is crucial for a collaborative effort to confront these issues. Details of triage tools and non-ED conveyance routes of care are insufficiently provided in study articles. Newton et al. [31] provide a detailed overview of referral routes, the accompanying triage tool and the accuracy of decision making in one UK ambulance trust; future studies should provide similar overviews, whilst expanding to assessment of patient outcomes.

As we aimed to identify non-ED conveyance alternatives that could be adapted to a wide range of intermediate patients, we excluded studies that provided specific clinical pathways; for example, community paramedicine pathways that provide proactive home visits, specific fall pathways or alcohol detoxification centres [73-75]. However, synthesis of the effectiveness of these targeted schemes is needed. A combination of specific clinical alternatives along with general pathways for intermediate patients can help ensure the highest number of patients can be referred to the optimal level of care.

Finally, the variability in study design, scheme, protocol, outcome and sample population rendered it difficult to synthesize and summarize the evidence. While several studies reported similar outcomes including decision accuracy matrices and recontact rates, heterogeneity of these outcomes made us unable to consider a formal meta-analysis. Additionally, several studies grouped patients who were not immediately conveyed to ED together; thus, it was not possible to assess if referrals or transfers to alternate care routes provide a better safety net than self-care at home.

\section{Future steps}

Given the widespread use of alternative schemes in the UK and Sweden, and increasing implementation in North American countries, it is crucial to commission large scale studies evaluate patient outcomes for those conveyed directly to ED, those left at home and those referred to alternate routes of care. Notwithstanding the impact of decreasing emergency department burden and cost effectiveness, patient safety must remain the most important outcome. Studies consider the entire patient journey, which may involve linkage of data from several emergency or primary care services. There must be improved collaboration between ambulance services within a single country and shared opportunities to learn from other countries. Given that schemes are linked to paramedic skill, training and education (e.g. research nurse, degree paramedic, emergency medical technician), the growing positive advancements in paramedic education in recent years may provide new opportunities and additional scope for these new patient pathways $[65,66]$.

\section{Conclusions}

This scoping review provided a broad summary of current evidence and consensus-based articles that examined alternate routes of care for the intermediate, non-urgent patients. Most evidence suggests that such schemes can improve operational efficiency, reduce ED conveyance and provide an optimal care pathway for the patient. Paramedics, GPs, patients and stakeholders all expressed a high willingness and recognised the benefits of such a scheme. Still, the majority of the studies lacked methodologically rigorous design and evidence of safe outcomes; there remains a significant need to examine patient safety in non-ED conveyance schemes.

\section{Appendix \\ Search strategy in PubMed}

1. paramedic*

2. ambulance*

3. "emergency service"*

4. "emergency medical service"*

5. "emergency technician"

6. "emergency practitioner"

7. "emergency dispatch"

8. "first responder"

9. "emergency rescue"

10. "emergency triage"

11. "emergency care practitioner" 


\section{1 OR 2 OR 3 OR 4 OR 5 OR 6 OR 7 OR 8 OR 9 OR 10 OR 11}

13. referral*

14. triage*

15. "emergency department"

16. "accident and emergency"

17. "A\&E"

18. "non convey":

19. non-convey"

20. deflect*

21. 13 OR 14 OR 15 OR 16 OR 17 OR 18 OR 19 OR 10

22. 12 AND 21

\section{Abbreviations}

ED: Emergency department; EMS: Emergency medical services; GP: General practitioner; PRISMA-SCR: Preferred Reporting Items for Systematic Reviews and Meta-Analyses extension for Scoping Reviews; RETTS: Rapid Emergency Triage and Treatment System; RCT: Randomised control trial; UK: United Kingdom; USA: United States of America

\section{Acknowledgements}

The authors are grateful to Matt Holland, librarian at NWAS NHS Trust, for his assistance in obtaining some of the more difficult full text articles and to Susan Rhind, paramedic at NWAS NHS Trust, who assisted with initial title and abstract screening.

\section{Authors' contributions}

$J M B, D J R, D R$ and KR contributed to study concept and design and acquisition of funding. JMB, DJR and EP contributed to acquisition of the data, analysis and interpretation of the data. JMB was responsible for drafting the manuscript. All authors critically revised the manuscript for important intellectual content.

\section{Funding}

This work was supported by the North West Ambulance Service.

\section{Availability of data and materials}

Data sharing is not applicable to this article as no datasets were generated or analysed during the current study.

\section{Ethics approval and consent to participate}

Not applicable.

\section{Consent for publication}

Not applicable.

\section{Competing interests}

The authors declare that they have no competing interests.

\section{Author details}

${ }^{1}$ MRC Unit for Lifelong Health and Ageing, UCL, 1-19 Torrington Place, London WC1E 7HB, UK. ${ }^{2}$ North West Ambulance Service, NHS Trust, Bolton, UK. ${ }^{3}$ Division of Geriatric Medicine, Department of Medicine, Dalhousie University, Halifax, Canada. ${ }^{4}$ Welsh Ambulance Services NHS Trust, Denbighshire, UK. ${ }^{5}$ Greater Manchester Health and Social Care Partnership, Manchester, UK.

Received: 9 September 2020 Accepted: 2 December 2020 Published online: 06 January 2021

\section{References}

1. Department of Health. Building the evidence base in pre-hospital urgent and emergency care: a review of research evidence and priorities for future research. 2010. Available at: https://www.gov.uk/government/publications/ building-the-evidence-base-in-pre-hospital-urgent-and-emergency-care-areview-of-research-evidence-and-priorities-for-future-research.
2. Scottish Ambulance Service, 2019. Scottish ambulance service annual report and accounts for year ended. Accessed 5 Aug 2020 at http://www. scottishambulance.com/userfiles/file/TheService/Annual\%20report/201906-26\%20Fina|\%20Board\%20approved\%20Annual\%20Accounts\%2018-19\%2 0v2.pdf.

3. Department of Health, 2019. Northern Ireland Hospital Statistics: Emergency Care 2018/19. Accessed 5 Aug 2020 at https:/www.health-ni.gov.uk/sites/ default/files/publications/health/hs-emergency-care-18-19_0.pdf.

4. NHS 2019. Ambulance Quality Indicators Data 2018-19. Accessed 5 Aug 2020 at https://www.england.nhs.uk/statistics/statistical-work-areas/ ambulance-quality-indicators/ambulance-quality-indicators-data-2018-19/.

5. Stats Wales, 2020. Emergency ambulance calls and responses to red calls, by LHB and year. Assessed 5 Aug 2020 at https://statswales.gov.wales/ Catalogue/Health-and-Social-Care/NHS-Performance/Ambulance-Services/ emergencyambulancecallsandresponsestoredcalls-by-lhb-year.

6. Department of Health. Tackling demand together: a toolkit for improving urgent and emergency care pathways by understanding increases in 999 demand. 2013. Available at: https://webarchive.nationalarchives.gov.uk/2013 0124051819/http://www.dh.gov.uk/prod_consum_dh/groups/dh_ digitalassets/documents/digitalasset/dh_106924.pdf.

7. NHS England. Transforming urgent and emergency care services in England. Urgent and emergency care review. End of phase 1 report. Available at: http://www.nhs.uk/NHSEngland/keogh-review/Documents/UECR.Ph1Report. FV.pdf. 2013.

8. Mayer GG, Villaire M, Connell J. Ten recommendations for reducing unnecessary emergency department visits. J Nurs Adm. 2005;35(10):428.

9. Improving emergency department access by using a regional dashboard for ambulance diversion. Emerg Med Australas. 2011;23(1):109-11.

10. Brown LH, Hubble MW, Cone DC, Millin MG, Schwartz B, Patterson PD, et al. Paramedic determination of medical necessity: a meta- analysis. Prehosp Emerg Care. 2009;13(4):516.

11. Hauswald M. Can paramedics safely decide which patients do not need ambulance transport or emergency department care? Prehosp Emerg Care. 2002;6(4):383-6.

12. Office NA. NHS Ambulance Services. 2017. https://www.nao.org.uk/wpcontent/uploads/2017/01/NHS-Ambulance-Services.pdf.

13. Blodgett JM, Robertson D, Radcliffe D, Rockwood K. Creating a safety net for patients in crisis: paramedic perspectives towards a GP referral scheme. J Paramed Pract. 2017;9(1):11-7.

14. Ebben RHA, Vloet LCM, Speijers RF, Tönjes NW, Loef J, Pelgrim T, et al. A patient-safety and professional perspective on non-conveyance in ambulance care: a systematic review. Scand J Trauma Resusc Emerg Med. 2017;25(1):71.

15. Gratton MC, Ellison SR, Hunt J, Ma OJ. Prospective determination of medical necessity for ambulance transport by paramedics. Prehosp Emerg Care. 2003; 7:466-9.

16. Blodgett JM, Robertson DJ, Ratcliffe D, Rockwood K. An alternative model of pre-hospital care for 999 patients who require non-emergency medical assistance. Intl J Emerg Serv. 2017;6(2):99-103.

17. Snooks HA, Kingston MR, Anthony RE, Russell IT. New models of emergency Prehospital care that avoid unnecessary conveyance to emergency department: translation of research evidence into practice? Sci World J. 2013;2013:182102.

18. Snooks HA, Dale J, Hartley-Sharpe C, Halter M. On-scene alternatives for emergency ambulance crews attending patients who do not need to travel to the accident and emergency department: a review of the literature. Emerg Med J. 2004;21(2):212-5.

19. Jensen JL, Carter AJ, Rose J, Visintini S, Bourdon E, Brown R, et al. Alternatives to traditional EMS dispatch and transport: a scoping review of reported outcomes. CJEM. 2015;17(5):532-50.

20. Agarwal G, McDonough B, Angeles R, Pirrie M, Marzanek F, McLeod B, et al. Rationale and methods of a multicentre randomised controlled trial of the effectiveness of a community health assessment Programme with emergency medical services (CHAP-EMS) implemented on residents aged 55 years and older in subsidised seniors' housing buildings in Ontario. Canada BMJ Open. 2015;5(6):e008110.

21. Arksey H, O'Malley L. Scoping studies: towards a methodological framework. Int J Soc Res Methodol. 2005;8(1):19-32.

22. Grant MJ, Booth A. A typology of reviews: an analysis of 14 review types and associated methodologies. Health Inf Libr J. 2009;26:91-108.

23. Tricco AC, Lillie E, Zarin W, et al. P. RISMA extension for scoping reviews (PRISMA-SCR): checklist and explanation. Ann Intern Med. 2018;169:467-73. 
24. Melnyk BM, Fineout-Overholt E. Box 1.3: Rating system for the hierarchy of evidence for intervention/treatment questions. Evidence-based practice in nursing \& healthcare: A guide to best practice. 3rd ed. Philadelphia: Wolters Kluwer Health; 2015. p. 11

25. Blodgett JM, Robertson DJ, Ratcliffe D, Rockwood K. Piloting data linkage in a prospective cohort study of a GP referral scheme to avoid unnecessary emergency department conveyance. BMC Emerg Med. 2020;20:48.

26. Ebben RHA, Castelijns M, Frenken J, Vloet LCM. Characteristics of nonconveyance ambulance runs: a retrospective study in the Netherlands. World J Emerg Med. 2019;10(4):239-43.

27. Krumperman K, Weiss S, Fullerton L. Two types of Prehospital systems interventions that triage low-acuity patients to alternative sites of care. South Med J. 2015;108(7):381-6.

28. Larsson G, Holmén A, Ziegert K. Early prehospital assessment of non-urgent patients and outcomes at the appropriate level of care: a prospective exploratory study. Int Emerg Nurs. 2017;32:45-9.

29. Magnusson C, Källenius C, Knutsson S, Herlitz J, Axelsson C. Pre-hospital assessment by a single responder: the Swedish ambulance nurse in a new role: a pilot study. Int Emerg Nurs. 2016;26:32-7.

30. Magnusson C, Herlitz J, Axelsson C. Patient characteristics, triage utilisation, level of care, and outcomes in an unselected adult patient population seen by the emergency medical services: a prospective observational study. BMC Emerg Med. 2020;20(1):7.

31. Newton M, Tunn E, Moses I, Ratcliffe D, Mackway-Jones K. Clinical navigation for beginners: the clinical utility and safety of the paramedic pathfinder. Emerg Med J. 2014;31(e1):29-34.

32. O'Cathain A, Knowles E, Bishop-Edwards L, Coster J, Crum A, Jacques R, et al. Understanding variation in ambulance service non-conveyance rates: a mixed methods study. Health Serv Deliv Res. 2018;6(19).

33. Pickstone N, Lee GA. Does the @home team reduce local Emergency Department attendances? The experience of one London service. Int Emerg Nurs. 2019;46:100774.

34. Schaefer RA, Rea TD, Plorde M, Peiguss K, Goldberg P, Murray JA. An emergency medical services program of alternate destination of patient care. Prehosp Emerg Care. 2002;6(3):309-14.

35. Schmidt T, Atcheson R, Federiuk C, Mann NC, Pinney T, Fuller D, et al. Evaluation of protocols allowing emergency medical technicians to determine need for treatment and transport. Acad Emerg Med. 2000;7(6):663-9.

36. Schmidt TA, Atcheson R, Federiuk C, Mann NC, Pinney T, Fuller D, et al. Hospital follow-up of patients categorized as not needing an ambulance using a set of emergency medical technician protocols. Prehosp Emerg Care. 2001;5(4):663-9.

37. Snooks H, Foster T, Nicholl J. Results of an evaluation of the effectiveness of triage and direct transportation to minor injuries units by ambulance crews. Emerg Med J. 2004;21(1):105-11.

38. Snooks H, Kearsley N, Dale J, Halter M, Redhead J, Cheung WY. Towards primary care for non-serious 999 callers: results of a controlled study of "treat and refer" protocols for ambulance crews. Qual Saf Health Care. 2004; 13(6):435-43.

39. Tohira H, Fatovich D, Williams TA, Bremner A, Arendts $G$, Rogers $\mid R$, et al. Which patients should be transported to the emergency department? A perpetual prehospital dilemma. Emerg Med Australas. 2016;28(6):647-53.

40. Verma AA, Klich J, Thurston A, Scantlebury J, Kiss A, Seddon G, et al. Paramedic-initiated home care referrals and use of home care and emergency medical services. Prehosp Emerg Care. 2018;22(3):379-84.

41. Vicente V, Svensson L, Wireklint Sundström B, Sjöstrand F, Castren M. Randomized controlled trial of a prehospital decision system by emergency medical services to ensure optimal treatment for older adults in Sweden. J Am Geriatr Soc. 2014;62(7):1281-7.

42. Villarreal M, Leach J, Ngianga-Bakwin K, Dale J. Can a partnership between general practitioners and ambulance services reduce conveyance to emergency care? Emerg Med J. 2017;34(7):459-65.

43. Brydges M, Spearen C, Birze A, Tavares W. A culture in transition: paramedic experiences with community referral programs. CJEM. 2017;17(6):631-8.

44. Bury $G$, Janes D, Dowling J. General practice out-of-hours co-operatives in Ireland-emergency service or not? Ir J Med Sci. 2005;174(3):47-52.

45. Höglund E, Schröder A, Möller M, Andersson-Hagiwara M, Ohlsson-Nevo E. The ambulance nurse experiences of non-conveying patients. J Clin Nurs. 2019;28(1-2):235-44

46. Jones CM, Wasserman EB, Li T, Shah MN. Acceptability of alternatives to traditional emergency care: patient characteristics, alternate transport modes, and alternate destinations. Prehosp Emerg Care. 2015;19(4):516-23.
47. Knowles E, Bishop-Edwards L, O'Cathain A. Exploring variation in how ambulance services address non-conveyance: a qualitative interview study. BMJ Open. 2018;8:e024228.

48. Lederman J, Löfvenmark C, Djärv T, Lindström V, Elmqvist C. Assessing nonconveyed patients in the ambulance service: a phenomenological interview study with Swedish ambulance clinicians. BMJ Open. 2019;9:e030203.

49. Miles J, Coster J, Jacques R. Using vignettes to assess the accuracy and rationale of paramedic decisions on conveyance to the emergency department. British Paramed J. 2019;4(1):6-13

50. Power B, Bury G, Ryan J. Stakeholder opinion on the proposal to introduce 'treat and referral' into the Irish emergency medical service. BMC Emerg Med. 2019;19(1):81.

51. Rantala A, Forsberg A, Ekwall A. Person-centred climate and psychometrical exploration of person-centredness and among patients not conveyed by the ambulance care service. Scand J Caring Sci. 2018:32(2):852-60.

52. Snooks HA, Kearsley N, Dale J, Halter M, Redhead J, Foster J. Gaps between policy, protocols and practice: a qualitative study of the views and practice of emergency ambulance staff concerning the care of patients with nonurgent needs. Qual Saf Health Care. 2005;14(4):251-7.

53. Vicente V, Castren M, Sjöstrand F, Sundström BW. Elderly patients' participation in emergency medical services when offered an alternative care pathway. Int J Qual Stud Health Well-being. 2013;8:20014.

54. Alpert A, Morganti KG, Margolis GS, Wasserman J, Kellermann AL. Giving EMS flexibility in transporting low-acuity patients could generate substantial Medicare savings. Health Aff (Millwood). 2013;32(12):2142-8.

55. Altoft L, Raven D. It takes two. Intermediate Care. 2003;113(5841):28-9.

56. Arendts G, Sim M, Johnston S, Brightwell R. ParaMED Home: a protocol for a randomised controlled trial of paramedic assessment and referral to access medical care at home. BMC Emerg Med. 2011;11:7.

57. Australian New Zealand Clinical Trials Registry. ACTRN12610001064099 Updated 2018, Available from: http://www.anzctr.org.au/Trial/Registration/ TrialReview.aspx?ID=335818

58. Asplin BR. Undertriage, overtriage, or no triage? In search of the unnecessary emergency department visit. Ann Emerg Med. 2001;38(3):282-5.

59. Committee EMS. Alternate ambulance transportation and destination. Ann Emerg Med. 2001;38(5):616.

60. American College of Emergency Physicians, National Association of EMS Physicians. Alternate ambulance transportation and destination. Ann Emerg Med. 2008;52(5):594

61. Hsiao AK, Hedges JR. Role of the emergency medical services system in region wide health monitoring and referral. Ann Emerg Med. 1993;22(11): 1696-702.

62. Morganti KG, Alpert A, Margolis G, Wasserman J, Kellermann AL. Should payment policy be changed to allow a wider range of EMS transport options? Ann Emerg Med. 2014;63(5):615-26.

63. Munjal KG, Margolis GS, Kellermann AL. Realignment of EMS reimbursement policy : new Hope for patient-centered out-of-hospital care. JAMA. 2019; 322(4):303-4.

64. Sawyer NT, Coburn JD. Community Paramedicine: 911 alternative destinations are a patient safety issue. West J Emerg Med. 2017;18(2):219-21.

65. Hou X-Y, Rego J, Service M. Paramedic education opportunities and challenges in Australia. Emerg Med Australas. 2013;25:114-9.

66. Petter J, Armitage E. Raising educational standards for the paramedic profession. J Paramed Pract. 2012;4(4):241-2.

67. Jensen JL, Travers AH, Marshall EG, Cain E, Leadlay S, Carter AJE. Insights into the implementation and operation of a novel paramedic long-term care program. Prehosp Emerg Care. 2014;18(1):86-91

68. Hariton E, Locascio JJ. Randomised controlled trials - the gold standard for effectiveness research: study design: randomised controlled trials. BJOG. 2018;125(13):1716.

69. Ball SL, Greenhalgh J, Roland M. Referral management centres as a means of reducing outpatients attendances: how do they work and what influences successful implementation and perceived effectiveness? BMC Family Pract. 2016;17(37).

70. Kuttner R. The American health care system. Health insurance coverage. N Engl J Med. 1999;340(2):163-8.

71. Ahl C, Hjälte L, Johansson C, Wireklint-Sundström B, Jonsson A, Suserud BO. Culture and care in the Swedish ambulance services. Emerg Nurse. 2005;13(8):30-6.

72. Wihlborg J, Edgren G, Johansson A, Sivberg B. The desired competence of the Swedish ambulance nurse according to the professionals - a Delphi study. Intl Emer Nurs. 2014;3:127-33. 
73. Smith-Bernardin SM, Kennel M, Yeh C. EMS can safely transport intoxicated patients to a sobering center as an alternate destination. Ann Emerg Med. 2019;74(1):112-8.

74. Snooks HA, Anthony R, Chatters R, et al. Paramedic assessment of older adults after falls, including community care referral pathway: cluster randomized trial. Ann Emerg Med. 2017;70(4):495-505.

75. Mikolaizak AS, Lord SR, Tiedemann A, et al. A multidisciplinary intervention to prevent subsequent falls and health service use following fall-related paramedic care: a randomised controlled trial. Age Ageing. 2017;46(2):200-7.

\section{Publisher's Note}

Springer Nature remains neutral with regard to jurisdictional claims in published maps and institutional affiliations.

Ready to submit your research? Choose BMC and benefit from:

- fast, convenient online submission

- thorough peer review by experienced researchers in your field

- rapid publication on acceptance

- support for research data, including large and complex data types

- gold Open Access which fosters wider collaboration and increased citations

- maximum visibility for your research: over 100M website views per year

At BMC, research is always in progress.

Learn more biomedcentral.com/submissions 